\title{
Facilitators and Barriers of Patient Centered Care Practice in Public Hospitals of Benishangul Gumuze Regional State, South West Ethiopia
}

\author{
Tamiru Bogale \\ Pawe Health Sciences College jointly with Bahirdar University, Pawe, Ethiopia
}

Email address:

tamiru730@gmail.com

\section{To cite this article:}

Tamiru Bogale. Facilitators and Barriers of Patient Centered Care Practice in Public Hospitals of Benishangul Gumuze Regional State, South West Ethiopia. Rehabilitation Science. Vol. 6, No. 1, 2021, pp. 10-19. doi: 10.11648/j.rs.20210601.12

Received: October 12, 2020; Accepted: October 26, 2020; Published: April 16, 2021

\begin{abstract}
Background: Patient centered care has become a central for the nation's healthcare system all over the world. However the major issues facing healthcare systems around the world was not well explored. Therefore this study aims to identify facilitator and barriers to implement patient centered care practice in public hospitals in Benishangul Gumuze regional state, South West Ethiopia, 2019. Methods: Institution based Qualitative case study design was employed. Thirty two purposively selected participants from different category were interviewed. Ethical clearance sheet was obtained from Pawe health science college ethical review board. Seventeen face to face in depth interview, two focused group interview and systematic observation was conducted. Deductive analysis was employed manually to analyze text data. Results: The analysis result yields multiple driving and hindering factors of patient centered care practice. Information related factor, service expansion, feedback mechanism and health policy were identified as facilitators. In contrast patients' health literacy level, health care provider characteristics, patient empowerment, shortage of resource, organizational capacity, shortage of specialty care, managerial skill, legislation related factor and organizational culture are identified as a barrier to practice patient centered care. Conclusion: Patient centered care practice in public hospitals had cheaply driven or hindered by multiple factors. Patient health literacy level, patient empowerment, and family and friend involvement in patient care were perceived as a tough barriers. Investing on the improvement of patient health literacy and patient empowerment may fasten the transformation of the current health care practice in to patient centered care practice in the healthcare settings.
\end{abstract}

Keywords: Patient Centered Care, Hospitals, Care, Patient, Ethiopia

\section{Background}

The principle of patient-centered care is date back to the ancient Greek, which was interested in the particulars of each patient. In the 1970s, it is coming to prominence. Patient-centered care is highly valued in the discipline of nursing. The origins of this value can be traced to Florence Nightingale, who differentiated nursing from medicine by its focus on the patient rather than the disease. Patient centered care (PCC) is a care that is holistic, empowering and that recognizes the individual's priorities and needs that important to all patients [1-5].

According to the Picker Institute, patient-centered care has eight delineated dimensions, including: respect for the patient's values, preferences, and expressed needs; information and education; access to care; emotional support to relieve fear and anxiety; involvement of family and friends; continuity and secure transition between health care settings; physical comfort; and coordination of care. PCC can improve quality of care and patient satisfaction, as well as reduce healthcare costs. Currently the approach gets the emphasis from health policy makers to make the health care system more efficient to achieve the need of the patient at the primary health care level and it has become a central for the nation's healthcare system all over the world [6-10].

The success of PCC is dependent on the strong partnerships between health care organizations, health care providers, and support service staff, as well as patients. Patient-centered care is associated with improved overall health status of patients, improved health care delivery efficiency, improved perception 
of satisfaction, increased meeting of expectations, and improved quality and service excellence. The outcome of having a patient- centric demonstrates that patients will be more engaged in their care, resulting in a strong positive impact on loyalty among consumers, a stronger competitive advantage, and results in more treatment compliance [11-17].

Despite proven benefits of patient Centered Care, significant challenges still persist in its implementation across the globe. Its integration into clinical practice is often found to be patchy and inconsistent even in developed countries. PCC in Africa is increasingly recognized as a key dimension of quality healthcare, but unfortunately remains poorly implemented in practice. Poorly implemented PCC in sub-Saharan region is contributed by three distinct factors; the performance of providers, the structural and organizational features of the health system and the broader socioeconomic environment in which health workers operate. Traditionally, provision of care in developing countries including Ethiopia has been organized around the needs and desires of healthcare professionals, particularly physicians [18-22].

As a consequence the provision of healthcare service in Ethiopia is more biomedical than bio- psychosocial. Study conducted in 2016 shows, $71 \%$ of healthcare providers admitted to failing to deliver compassionate and respectful care services and $77 \%$ of them acknowledged that they did not deliver compassionate and respectful care for every patient they encounter. Another study conducted in 2015 shows, patient satisfaction is not adequate and still $30 \%$ of patients at in- patient service were not satisfied by the services they received. The baseline assessment of the Ethiopian Hospitals Reform Implementation in some hospitals of the country also shows, the nursing standard of key performance indicators were still unmet and a system for coordinating and managing nursing staff was partially completed. This means out of the eight performance indicators for nursing care standard, only one has been partiallycompleted, resulting in an overall compliance rate of $12.5 \%$ [23-25].

As the demand of the patient health need in the health care industry is growing, patient centered care is considered to be crucial for a health care organization that provides caring, safe and high quality care and is described as holistic, non-judgmental, empathetic, respectful, and empowering. The achievement of patient centered care is indicated by service satisfaction of the patient. Improvement in the quality of hospital care is a fundamental aspect of health system strengthening that is directly linked to the service delivery dimension of the World HealthOrganization (WHO) building blocks of a health system. Ethiopia's implementation of a national system for monitoring hospital performance is proofed as an example of a low-income country that aims to improve health service delivery via the creation of a culture of accountability and decentralization [26-30].

Currently, the Ethiopian Hospital Services Transformation Guideline, which is consistent with the national focus on quality improvement in health care, which are also consistent with the Health Sector Transformation Plan. Subsequently, the country developed a hospital performance monitoring system based on achievement of key performance indicators and the Ethiopia Hospital Alliance for Quality. The first focus area of the EHAQ is patient satisfaction to measure the performance of hospitals across the country including labour and delivery quality [31-36]. Therefore the study was focused on exploring the facilitators and barriers of patient centered care practice in public hospitals, by using manager's, health care provider's, supportive staff and patients' perspective at the health care organization level.

\section{Methods and Materials}

The study was conducted in two public hospitals in Benishangul Gumuze regional state, South West Ethiopia, from October 1/2019 to December 30/2019.

Assosa general hospital is one of the public hospitals in the region, which is situated in Assosa town. The hospital was established in 1977 E.C and renovated in 1982 E.C. Pawe general hospital is one of the public hospitals in the region, which is situated in Pawe town, Metekle zone. The hospital was established in 1980 E.C.

Institution based qualitative case study design was employed because this study seeks to provide a rich understanding of the study phenomenon contextually. A sample that best represents the diverse source of information was taken purpusively and opinions of those potential sources of information (52) was considered when choosing participants from both organizations. Therefore 32 participants from different category (health care providers, patients and administrative staff) were selected purposively. Two general managers (CEO), seven health care providers (two nurse, two midwifery, one pharmacy, one physician, and one Laboratory), four supportive staffs and four patients, totally thirteen staffs and four patients were participated in in-depth interview and fifteen health care providers were participated in focused group discussion. Data was collected by using multi-method strategy, which consists; individual interview, focused group interview, observation and document review. Deductive analysis was employed manually based on predetermined framework. Looking and reading of translated text data and field note was made repeatedly to familiarize with data. Different text highlight color was used to select similar concepts from the text data and to generate initial codes. Quotes were extracted from the text data parallary and stored separately.

Similar coded datum was assembled together. After conceptualizing of related coded datum, searching of categories among codes was made. Then initial category was made. After reviewing the initial categories, categorizing of the categories into predetermined theme was done. Finally interview result was written by compiling memos, quotes and analyzed data. The observational data of the three observers was tallying up on each item of structured observation check list on the column of fully practiced, partially practiced or not practiced. Then the observation result was analyzed qualitatively by item level using category system as fully practiced, partially practiced or not practiced and the result 
was written based on each section of the structured observation checklist. Finally, the interview results, observation results and document review result were triangulated.

The rigor of the work was maintained by different operational techniques: collecting rich data by careful interview of individuals and groups from heterogeneous participants, and using multi-method strategy of data collectionwhich consists; systematic observation and reviewing of the interview guide by experts. Member check of the analysis result was made to check that the analysis result is exactly as participants' experiences before writing of final result report by inviting to read the translated data for staff participants. All interviews was tape-recorded and noted on hard copy to provide back up. All data was stored by the principal investigator on pass word protected computer and hard copy in private room. Prior to data collection Ethical clearance was obtained from Pawe health science college ethical review board.

\section{Results}

\subsection{Theme One: Facilitators to Practice Patient Centered Care}

Under this theme factors that drive the patient centered care practice in healthcare organizations were described. Informational factor, stakeholder and partner support, service expansion, monitoring and feedback mechanism, national policy and team spirit are perceived as a facilitating factor to practice patient centered care in public hospitals of the region.

\subsubsection{Informational Factors}

As most respondent's experience shows, information is everything. Accessibility of care without information is impossible; continuity of care without information is impossible; involving the patient without information is nothing. Patient centered care practice without patient empowerment is impossible. System for information flow within the organization and outside the organization is in place to enhance the provision of health care. Scheduled health information provision is tried to practice in hospitals and information desk is available. Supportive staff participant two said that:

"[...] health information is provided every morning from Monday to Friday and written directions for most service unit are there to guide the patient" (Supportive staff participant 2).

\subsubsection{Stakeholder and Partner Support}

As some respondent opinion, establishment of new supplier branch at near distance is considered as a driving factor to practice patient centered care. Health care provider participant six said that: "[......] the establishment of Assosa branch pharmaceutical and fund supply agency (PFSA) may solve shortage of medical supplies in our hospital [......]" (Health care provider participant 6 ). In the other hand, workings with different partners make an opportunity for their hospitals to mobilize resources. Health care provider participant three said that:

"[.....] this hospital has an opportunity to get different equipment from the partners; we have got different costly medical equipment that are not easily purchased by our hospital capacity, for example, one of our partners donates different equipment for establishment of our medical ICU and neonatal ICU' (Health care provider participant3). By few health care provider respondents, human power is considered as a factor that can drive the patient centered care practice. Focused group discussion participant eight said that:

"[...] if we talk frankly, the number of physician currently is about twenty but previously it was not more than two, now there are enough midwives, integrated emergency surgery and obstetrics (IESO) and enough nurses"(FGD1P8).

\subsubsection{Service Expansion}

Plantation of advanced diagnostic machine like gene $\mathrm{x}$-pert and CD4 and starting of RH compatibility test in health care organization level is considered as a driving factor to practice patient centered care. Health care provider participant one said that:

"[...] plantation of gene $x$-pert machine in this hospital will strengthen the diagnosis of MDR TB and it is also used as referral diagnostic center for other catchment health centers" (Health care provider participant1).

\subsubsection{Monitoring and Feedback Mechanism}

Monitoring system is placed to follow the clinical practice of inpatients in their hospital as all manager and some of health care provider respondent explained. Of course, morning session is a kind of clinical audit, which includes a group of people from different professions and from different service units. It is also considered as a method of making clinical solution for the problems happened in the hospital. Therefore it has contribution for improvement of patient health if it is done with commitment and Manager respondent two said that:

"[...] there is a morning session conducted in our hospital three times per a week, Monday, Wednesday and Friday" (Manager Participant 2).

Most manager and health care provider respondents believed that feedback from the patient in the organization, the community forum and the staff forum is taken to use as an input to improve quality of service in their hospitals. Most of the feedback focuses on the improvement of service quality and health care provider behaviors. As most respondent noted, any patient who have compliant have the right to submit his/her case for hospital by person or by putting the paper on the feedback box. Patient satisfaction survey is also conducted every quarter, in which communication, patient preference and desire need is included. It is clear that all this input is used for improvement of the quality of the service. Focused group discussion one participant seven said that:

"Community forum and staff forum is conducted every quarter throughout the year, this is an opportunity to receive feedback from them to improve the quality of service in our hospital" (FGD1P7). 


\subsubsection{National Health Policy}

The starting of Ethiopian hospital reform implementation guideline (EHRIG) before five year in the hospital is reported as a driving factor to practice patient centered care. As manager respondents, most of the dimension of EHRIG focuses on the leadership, environment of care, infection prevention, human resource...etc; generally it has thirteen chapters on different dimensions. They also believe that, EHRIG is used as framework to assess the performance of their hospital. What we learn from this is improving the hospital performance based on EHRIG standards means making the foundation for patient centered care practice. Currently EHRIG is expanding to Ethiopian hospital services transformation guide line (EHSTG). Manager respondent two said that:

"Ethiopian hospital reform implementation guideline was started before five years, [...], we are using this guideline as a framework to improve our hospital performance, currently it is replaced by Ethiopian hospital services transformation guide line"(Manager participant 2).

Following the implementation of different national policies in public hospitals, improvement is made on health service delivery. Medical records management system is improved and considered as driving factor for practicing patient centered care in the hospital as majority of respondent says. Focused group discussion one participant six said that:

"This time there is a change in this hospital, the medical registration number of the patient is retrieved by their name easily from the data base, previously if patient misses his/her medical registration number, they may reregistered as a new or to leave the hospital'(FGD1P6).

As all manager respondents, this time public hospitals are governed by board, there is a system to raise internal revenue of the hospital and they belief that the implementation of EHRIG in their hospital had made improvement on the quality of services. Manager participant one said that:

"[......] our hospital is governed by board; the revenue of the hospital is raised time to time. This is an opportunity to provide quality service" (Manager Participant 1).

\subsubsection{Team Spirit}

As few health care provider respondent, team spirit is considered as a driving factor in some service units to practice patient centered care in hospitals. It is obvious that, team spirit is more consumed by health care delivery system for integration, continuity and coordination of services in the health care organizations. Intra service unit team spirit is common than inter service unit team spirit. Health care provider participant five said that:

"[.....] the team spirit in the pharmacy department is good, in this service unit there are committed staffs that work with spirit for the improvement of patient satisfaction"'(Health care provider participant 5).

Furthermore, one to five networking is considered as team spirit. It premises the free discussion about daily activities, by this the strengths and weakness of the service unit is identified on daily basis. In this regard manager participant one said that:
"One to five networking is there at every service unit; this is important to strengthen group relationship in our service as well as to improve the quality of service [...]" (Manager Participant 1).

\subsection{Theme Two: Barriers to Practice Patient Centered Care}

Under this theme factors that hinder the patient centered care practice in healthcare organizations were described. Patient characteristics, health care provider characteristics, lack of patient empowerment, shortage of resource, organizational capacity, shortage of specialty care, managerial skill, legislation related factor and organizational culture are identified as a barrier that hinder patient centered care practice.

\subsubsection{Patients' Health Literacy Level}

Some of the staff respondents believed that low patient awareness regarding health information is considered as a hindering factor to practice patient centered care in their hospitals. Health care provider respondent seven said that:

"To practice patient centered care in our hospital, the patient awareness should be improved regarding health information. Without improvement of patient awareness, practicing patient centered care is challenging in our context" (Health care provider participant 7).

In the other view, improving basic education as well patient health literacy is considered as a core activity at the grass root level, at the community level to achieve patient centered care practice. Health care provider respondent four said that:

"If you want to practice patient centered care in this hospital, first the community awareness about health should be increased, then we can practice patient centered care [...], otherwise it is difficult"(Health care provider participant 4).

\subsubsection{Health Care Provider Characteristics}

As most health care provider participants, capacity building and commitment related factors are the most frequently listed health care provider related factors that hinder the practice of patient centered care in hospitals.

The need of capacity building for health care provider should not limited on patient centered care only. It should also address communication skill as well as ethical aspect of the health care. Not providing patient centered care training for the staff makes it complex to practicing patient centered care. Coaching of the newly deployed staffs by senior staff is also another approach of staff capacity building but it is not practiced widely in the health care organization. Health care provider respondent one said that:

"There is no coaching system in our service unit for newly deployed staffs; if it is applied, it will improve the capacity of the newly deployed staff" (Health care provider participant 1).

Attitude related factor is the other health care provider related factors that hindering patient centered care practice in hospitals. Health care provider respondent three said that:

"Some health care provider does not respect the patient and their family while they provide care [......]" (Health care provider participant 3 ). 
Commitment deficit from health care provider is reported by some focused group discussion participants as a hindering factor to practice patient centered care in their hospital. Focused group discussion one participant six said that:

"There was a situation that was happening due to the negligence of health care providers at ART clinic. There was one infant lost, because the family was not properly counseled and no full address was taken [......]" (FGD1P3).

As few focused group discussion participants noted that, motivational factor is also reported as hindering factor to practice patient centered care in their hospital. Focused group discussion one participant two said that:

"If we do have incentives, we can serve our patient with commitment, but no hardship incentive, no professional fee and no risk fee for health care providers"(FGD1P2).

\subsubsection{Lack of Patient Empowerment}

As most respondents report, limited desire to use information on the patient side and lack of clear and specific health information provision on the health care provider side is a bipolar problem for not to empowering the patient regarding their health status and their treatment option. As supportive staff respondent one said that:

"Our patients have no a culture to ask what they did not know and utilization of health information is low; there is a need to improve the level of patient awareness" (Supportive staff participant 1).

Patient education material for varying literacy level with local languages is not readily available for common health problems of the area. The patient contribution for their health is not well invested. The current health care paradigm needs the partnership of the patient. Without this action, achievement of patient centered care practice is impossible.

\subsubsection{Shortage of Resource}

Shortage of pharmaceutical supply and medical equipment, shortage of building and shortage of senior health care provider (specialists) are the most frequently listed resource related factors that hinder the patient centered care practice in hospital settings.

I. Pharmaceutical supply and medical equipment: Slowness of supply chain system plus limited supplies from the suppliers in amount and in kind makes it complex. Most of the respondents believed that shortage of medical supplies and drugs are the nationwide problem. The experience of health care provider respondent two and seven more strengthen this idea:

"[.....] the system that placed to deliver drug and medical supply in this hospital is too much slow between the requesting time and delivering time" (Health care provider participant 2). "Shortage of supplies and medical equipment is a big challenge in our country; it is challenging to practice patient centered care in our hospital even for the future" (Health care provider participant 7).

Providing quality of health service is affected by different factors. But shortage of pharmaceutical supplies is putted by the respondent at higher level. Supportive staff respondent three said that:
"Without adequate drug supply the patient may not satisfied by the received care from this hospital, this time the only chance for our patient is collecting the prescribed drugs from the private pharmacy"(Supportive staff participant 3).

Unavailable pharmaceutical supplies and laboratory services from the public health facility are usually complemented by private health facilities. But its negative impact is exposing the patient to unnecessary cost. Different context affects the accessibility of health services for the consumers. But socioeconomic context is the commonest one in our country. Patient respondent three was one of the victims and he said that:

"[...] for example the cost of the renal function test in this hospital is 40 birr and but its cost at private health facility may not be below 200 birr [....], we have exposed to unnecessary financial resource" (Patient participant3).

Despite shortage of pharmaceutical supplies and medical equipment, maintenance of nonfunctional equipment is not common in the hospital. As some respondents noted that, looking the crowded store by nonfunctional medical equipment is common in their hospitals. Focused group discussion two participant seven said that:

"Nonfunctional medical equipment is crowding the hospital store, no maintenance for all this nonfunctional equipment [..... "'(FGD2P7).

II. Infrastructure: Shortage of space for work environment is considered as resource related barrier which hinder patient centered care practice. Adequate space and well building design is prominent for health care delivery practice. Making the hospital building attractive in design and attractive compound is the first steps to maintain the patient's comfort and expectation. Efforts to make green compound is seen in health care organizations. But its attractiveness in one of the two hospitals is shadowed by the building design. In the other side, inadequate space is the problem of most service units in the public hospital. Focused group discussion one participant three said that:

"[......] there is space shortage at surgical ward, outpatient department, and other service units" (FGD1P3).

III. Human power: Shortage of specialized senior health care provider and turnover of the experienced health care providers is a practical challenge in a public hospital to practice patient centered care as explained by some health care provider and manager respondents. The manager respondent two explained his experience as:

"[...] turnover of the health care provider especially physician before two year work experience is common, they leave before finishing their assignments, it is the first barrier to practice patient centered care in our hospital'(Manager participant 2).

\subsubsection{Organizational Capacity}

Auditable pharmaceutical transaction and system (APTS) is a current evidence based practice to fasten drug information system and the service provision in one room. It was applied in different hospitals across our country. It is capacity related barrier, but shortage of buildings in all 
hospital's pharmacy service makes the problem more complex. It is frequently listed barriers by most managers and health care provider respondents. Health care provider respondent two said that:

"Auditable pharmaceutical transaction system (APTS) is still not established, it is beyond our capacity, support is requested from regional health bureau to establish it in our hospital' (Health care provider participant2).

\subsubsection{Shortage of Specialty Care}

The location of service unit and shortage of specialty services in the health care organization makes a difficult for the continuity of care. The location of some service unit is not suitable to provide coordinated health services. Health care provider respondent seven said that:

"The service units are not settled around one area, in our case those patients who have co morbidity receive care for only their priority health problems, for other problem may be on second day"(Health care provider participant 7).

Most of the respondents believe that, available specialty services are limited to secure the continuity of care within the organization. Providing referral sheet is common due to shortage of specialty services in the hospital. Focused group discussion one participant three said that:

"[...] there is no internist at medical ward; there is no pediatrician at pediatric ward and there is no radiologist at radiology department, there is a need of specialty service in this hospital [...]" (FGD1P3).

Furthermore, the turnover of specialists in the health care organization is a practical challenge to provide specialty care. Health care provider respondent three said that:

"[...] the deployed specialists in this hospital is almost none, there is turnover, even sponsored physicians" (Health care provider participant 3 ).

\subsubsection{Managerial Skill}

Most of the interviewee report touches, slow response for service related question and delayed decision for service related problem is a common manager related factors that hinder patient centered practice in their hospital. There is also a problem on doing as a team. Health care provider respondent one said that.

"On management side, there is a gap on doing as team, I know there is regular management committee meeting but they do not visit us, they do not ask our problems, even they do not respond our question timely"(Health care provider participant 1).

Distributed decision is considered as a motivational factor for hospital staffs. Some of the health care provider participants believed that, most of their leaders are stand at the side of the patient and they believe that, it has a negative impact on the health care provider's motivation. Health care provider respondent seven said that:

"[...], the manager is stands only for the right of the patient by neglecting the health care provider's right. In our hospital, there is a tendency that focuses on one side, the perception of the manager on patient's right is not good for me" (Health care provider participant7).
Monitoring and evaluating of the work in an organization is essential for performance improvement by in placing feedback mechanism system from patients and the community level. Patient satisfaction survey was conducted quarterly and system was in place to prepare plan and change in to action based on the survey results. Changing this into action is on up and down motion. Some of the staff respondent experience strengthens the above idea. Health care provider respondent one said that:

"[...] at the management side the hospital managers are looking the performance of the hospital carelessly, they are not monitored routinely, most of the hindering factors are persisted by the carelessness of the hospital managers" (Health care provider participant1).

Furthermore, one manager respondent noted that lack of clinical managerial skill at different service unit is considered as a leadership related barrier which hinders patient centered practice: "Leadership skills of ward heads in our hospital are different, which varies from one ward to anther ward, some of them have limitation of managerial skill" (Manager Participant 1).

\subsubsection{Legislation Related Factor}

Legislation related factor is discussed by most supportive staff participants. Unavailability of legislation related to the rights and obligations of the patient and the health care providers and restriction of visiting time for inpatient is considered as legislation related barriers. In principle visiting schedule of hospital for visitors should be for 24 hour. This principle is lacked in the health care organizations. Supportive staff respondent four said that:

"[...] visiting schedule should be flexible, those visitors coming from distant area was disappointed due to this inflexible visiting schedule" (Supportive staff participant 4).

There is no legislation regarding the patient's and the health care provider's rights and responsibilities in the health care organization. Without legal legislation accomplishing patient care with accountability and responsibility from the health care provider is impossible and the same is true for the patient. But principles that extracted from different national guidelines are available in peace of written materials and posted at different areas. Non legislative accountability is not effective as legislative accountability of health care provider for the patients. Supportive staff respondent two said that:

"Policy document regarding the rights and obligations of the patient and the health care provider is not known by our service unit, but different written information on this issue Specially the right and obligation of the patient is posted"(Supportive staff participant 2).

\subsubsection{Organizational Culture}

As the manager respondents, the organizations are committed to communicate the value as well as the vision of the organization to the staff and the community. The method of communicating the values and visions of the organization is commonly used quarterly community forum and staff forum, and by standing the banner from visible place in the organization. The manager respondent one said that: 
"The mission and vision of our hospital is posted on written materials at visible place where publics are easily read it and for those who can't read, it can be communicated during community meeting"(Manager participant 2).

Of course communicating the values and the vision of the organization is not an easy task, but communicating it every three month is not fair. It needs investment of time and development of strategy to make a picture in the mind of the provider and the consumer in a daily basis as a routine activity. Otherwise the leader can't pull the provider and the consumer rather than push them by his/her vision.

Table 1. Summary of themes, subthemes and main quotes of the analysis.

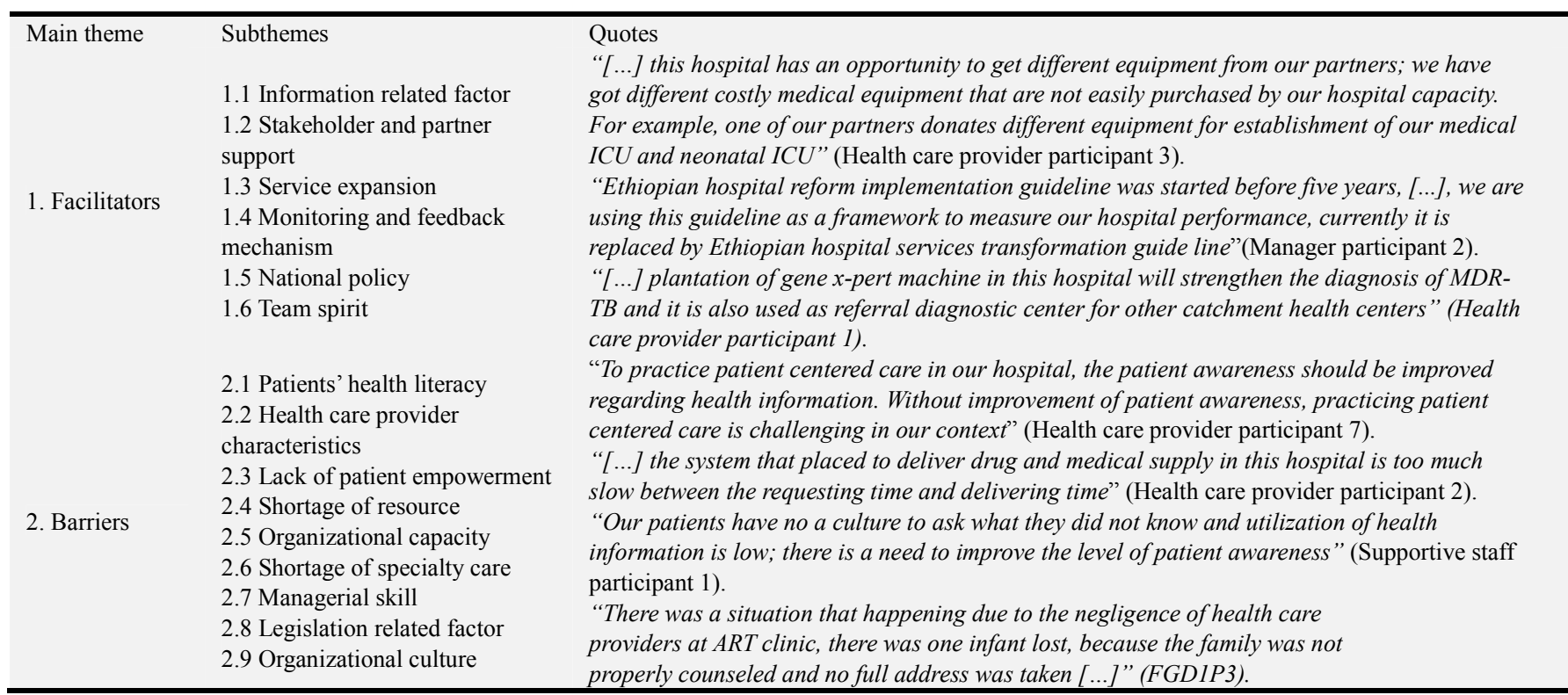

\section{Discussion}

In Ethiopia, patient centered care is a component of compassionate and respectful care (CRC) agenda in the health sector transformation plan since 2015. However the inclusiveness of patient centered care as a quality dimension in the health delivery system was date back to 2010. Based on the analysis of the text data facilitators and barriersof patient centered care practice was identified. This case study demonstrates how patient centered care practice is driven or hindered by multiple factors as perceived by the manager, the health care provider, supportive staff and patients.

Information related factor, stakeholder and partner support, service expansion, monitoring and feedback mechanism, and national policy were identified by this study as a facilitating factor to practice patient centered care in healthcare organizations. Some study report shows that, strong, committed senior leadership; clear communication of strategic vision; active engagement of patient and families throughout the institution; sustained focus on staff satisfaction; active measurement and feedback reporting of patient experiences; adequate resourcing of care delivery redesign; staff capacity building, and accountability and incentives were identified as a facilitators of patient centered care [46-50]. Information related factor in this data is richer than the previous studies. In the other hand, clear communication of strategic vision, active engagement of patient and families throughout the institution; sustained focus on staff satisfaction, accountability and incentives are more richer in the data of previous studies than the data of this study. The other study conducted in Republic of South Africa noted that factors which facilitate patient-centered care were a positive work environment for staff, demonstrating exemplary professional leadership, continuous in-service education for staff and collaborative teamwork within the interdisciplinary team [37]. Except team work, the data content of this study is also missed the data content of the previous study. But most of the factors that missed as a facilitator was included as barrier for practicing patient centered care in this study. It implies that the capacity of the staff and the leader in the setting of the previous study and the setting of this study is found in different level. This may be due to the socioeconomic context of the setting.

In this study experience of respondent shows that, patients' health literacy level, health care provider characteristics, lack of patient empowerment, shortage of resource, organizational capacity, shortage of specialty care, managerial skill, legislation related factor and organizational culture were identified as a barrier to practice patient centered care. But resource related barrier and patients' health literacy level is the most frequently listed barriers by majority of respondents. The study conducted in USA shows that changing the organizational culture from a 'provider-focus' to a 'patient-focus' and the length of time it took to transition toward such a focus were the principal barriers for patient-centered care practice [47]. This study data contains multiple factors than the previous study that identified as a barrier of patient centered care practice. However distribution of power and resistance to change is consisted richer in the data of previous studies than the data of this study [54]. 
Other qualitative study conducted in different country reveled that; traditional practices and structures; health care providers attitude; lack of adequate resource and increased administrative work were identified as barriers to practice patient centered care [36-45]. Pelzang by his literature review noted that lack of clear definition of patient centered care, inadequate educational emphasis on patient centered care, lack of coordination, integration and continuity of care, shortage of staff and dominance of biomedical health care are identified as barriers that hinder patient centered care practice [51-53]. Lack of adequate resource and healthcare provider's attitude is the content of the data in both the previous study and this study. Organizational structure, lack educational emphasis on patient centered care, increased administrative work and lack of clear definition of patient centered care were the factors that found richer in the data of the previous studies than this study. However lack of patient empowerment, patient literacy, lack of managerial skill, organizational capacity, organizational culture, legislation related factors and shortage of specialty care were identified factors that richer in the data content of this study than previous studies. A regional qualitative study conducted in sub Saharan African countries shows that, the training of health workers, the structural and organizational features of the health system and the broader socioeconomic environment in which health workers operate were identified as barriers to practice patient centered care in the health care organizations [18]. Two factors in the data content of this study were supported by the data content of the previous study. Still the managerial capacity, staff capacity, organizational capacity and shortage of resource take a great share on this regard to transform the existed healthcare delivery in to patient centered care in the public hospitals.

\section{Conclusion}

Patient centered care practice in public hospitals had cheaply driven or hindered by multiple factors. Patient health literacy level, patient empowerment, and family and friend involvement in patient care were perceived as a tough barriers. Information related factor, stakeholder and partner support, service expansion, monitoring and feedback mechanism, and national policy were identified as perceived facilitators. In contrast patients' health literacy level, health care provider characteristics, patient empowerment, shortage of resource, organizational capacity, shortage of specialty care, managerial skill, legislation related factor and organizational culture were identified as a barrier to practice patient centered care in public hospitals of the region. Optimizing the pharmaceutical supplies and equipment at the level ofservice demand is vital to prevent negative patient experience to the given health services in the healthcare organization, strengthening primary care and the primary care workforce would improve patient empowerment and decrease unnecessary patient load at the general public hospitals and optimizing the health literacy of the community at grass root level can promote patient empowerment; in turn it can improve patient and family involvement in decision making of their treatment option.

\section{List of Abbreviations}

APTS-Auditable pharmaceutical transaction and service

PCC-Patient centered care

CRC-Copassionate and respectfull care

EHSTG-Ethiopian hospital services transformation guide line

EHRIG-Ethiopian hospital reform implementation guideline

FGD-Focused group discussion

EHAQ-Ethiopia Hospital Alliance for Quality.

E.C-Ethiopian calander

CEO-Chief Excutive officer

\section{Declarations}

\section{Ethics Approval and Consent to Participate}

Ethical approval obtained from the ethical review committee of Pawe health Science College. Administrative permissions granted and confidentiality ensured using codes and keeping questionnaires locked. All respondents assured that the data would not have any negative consequence on any aspects of their life and participants who refused the consent respected.

\section{Consent for Publication}

Not applicable.

\section{Availability of Data and Material}

Additional file: Data abstraction tool.

\section{Competing Interests}

The authors have declared that no competing interest.

\section{Authors' Contribution}

Mr. Tamiru Bogale involved in the research concept of the study, planning field work analysis and interpretation, and manuscript write-up.

\section{Acknowledgements}

My thanks go to Pawe general hospital and Assosa general hospital staffs as well as patients from both hospitals for providing their experience during interview. Finally yet importantly I would like to thank Bahirdar University and Pawe health Science College for giving me the chance to conduct this study.

\section{References}

[1] Stewart Met al. The impact of patient-centered care on outcomes. The Journal of Family Medicine. 2000; 49 (9): 796-804.

[2] Lauver DR. Patient-Centered Interventions. Research in Nursing \& Health. 2002; 25: 246-55. 
[3] Morgan S. A Concept Analysis of Person-Centered CareJournal of holistic nursing. July 2011.

[4] Lusk JM. A Concept Analysis of Patient-Centered Care. Nursing Forum. 2013; 48 (2).

[5] Cance TM. Using the caring dimensions inventory as an indicator of person centered nursing. Journal of Clinical Nursing. 2011; 18: 409-17.

[6] Practitioners RCoG. An Inquiry into Patient Centered Care in the 21 st Century 2014.

[7] Shaller D. Patient-centered care: what does it take? Consulting S, editor 2007.

[8] Framptonetal. Patient-Centered Care Improvement Guide: Picker Institute and Planetree; October 2008.

[9] Alharbi. TSJ. The impact of organizational culture on the outcome of hospital care: After the implementation of person-centered care. Scandinavian Journal of Public Health. 2014; 42: 104-10.

[10] Alharbi. TSJ. Organizational culture and the implementation of person centered care: Results from a change process in Swedish hospital care. Health Policy. 2012; 108: 294-301.

[11] TFDRoEMoH. Health Sector Transformation Plan (HSTP) 2015/16 - 2019/20 (2008-2012 EFY). Ethiopia 2015.

[12] Balik B. Leaders' role in patient experience: Hospital leadership must drive efforts to better meet patients' needs. Healthcare Executive. 2011; 26 (4): 76-8.

[13] Abraham M. implementing patient- and family-centered care: Part 1: Understanding the challenges. Pediatric Nursing. 2012; 38 (2): 44-7.

[14] Epstein RM. Why the nation needs a policy Push on patient-centered health care. Health Affairs (Millwood). 2010; 29 (8): 1489-95.

[15] Natale CVea. The role of engaged patients. Healthcare Financial Management. 2013; 67 (8): 90-7.

[16] Davis. A 2020 Vision of Patient-Centered Primary Care. J GEN INTERN MED. 2005; 20: 953-7.

[17] JE M. Introduction to Person-centered Medicine: From Concepts to Practice. Journal of Evaluation in Clinical Practice. 2011; 17: 330-2.

[18] Medicine Io. Crossing the Quality Chasm: A New Health System for the 21st Century. Washington, DC: National Academy Press; 2001.

[19] De Boer D. The importance of patient-centered care for various patient groups. Patient Education and Counseling. 2013; 90: 405-10.

[20] Canada TCoFPo. Best advice -Patient-centered care in a Patient's Medical Home 2014.

[21] Man JD. Patient-Centered Care and People-Centered Health Systems in Sub-Saharan Africa: Why So Little of Something So Badly Needed? The international journal of patient centered medicine. 2016; Vol 6 (3).

[22] Serkalem Girma et al. An agenda for developing compassionate, respectful care and caring health care professionals. Special bulletin of Federal ministry of health
Ethiopia. 2016. (18 ${ }^{\text {th }}$ annual review meeting): 17.

[23] W/yohanesetal. Perceived patient satisfaction with in patient services at Jimma University Specialized Hospital, Southwest Ethiopia. BMC Res Notes. 2015; 8: 285-93.

[24] TadesseDMetal. Improvement in Adherence to Ethiopian Hospitals Reform Implementation Guideline nursing standards in Asella Referral Teaching Hospital: A Pre-post Study. Health systems and policy research. 2015; 2 (1: 14): 1-6.

[25] BerwickDM. Lessons from developing nations on improving health care. BMJ. 2004 May 8; 328 (7448): 1124-9.

[26] McCannonCJetal. The science of large-scale change in global health. JAMA. 2007 Oct 24; 298 (16): 1937-9.

[27] WHO. The WHO health systems framework. World Health Organization Regional Office for the Western Pacific, 2014.

[28] McNattetal. Z. A national system for monitoring the performance of hospitals in Ethiopia. Bulletin of the World Health Organization; Policy \& practice. 2015.

[29] FMOH. National hospital reform implementation guidelines. Ethiopian Hospital Management Initiative. January 2010.

[30] FMOH. Ethiopian health services transformation guidelines. Ethiopian hospital Management initiative. September 2016.

[31] MedicalServicesDirectorate. Hospital leaders in pursuit of quality. Ethiopian Healthcare Today. January 2012.

[32] Michael J. Barry. Shared Decision Making: The Pinnacle of Patient-Centered Care. N Eng J Med March 1, 2012.; 366 (9).

[33] Christine B. "If you build it, Will they come? Designing Truly Patient-Centered Health Care". Health Affairs (Millwood). 2011.

[34] Robbins DA, \& Curro, F. A. Defining Patient Centricity: Opportunities, Challenges, and Implications for Clinical Care and Research. Therapeutic Innovation \& Regulatory Science. 2013.

[35] IAPO. What is patient centered care? Review definitions and principles. second ed. Netherland, 2007.

[36] Baboo et al. Perceptions of patient-centered care at public hospitals in Nelson Mandela Bay. HEALT SA GESOND HEID 2016; 21: 397-405.

[37] Constandetal. Scoping review of patient-centered care approaches in healthcare. BMC Health Services Research. 2014; 14: 271.

[38] JanetMetal. A Concept Analysis of Patient-Centered Care. Nursing Forum. 2013; 48 (2).

[39] A. AD-Z. Promoting patient care: work engagement as a mediator between ward service climate and patient-centred care. Journal of Advanced Nursing. 2012; 68 (6): 1276-87.

[40] EpsteinMet al. Measuring patient centered communication in patient-physician consultations: theoretical and practical issues. Social Science \& Medicine. 2005; 61: 1516-28.

[41] Postetal. The other half of the whole: teaching patients to communicate with physicians. Family Medicine. 2002; 34: 344-52. 
[42] Griffinetal. Effect on outcomes of intervention to alter the interaction between patients and practitioners: a systematic review of trial. Annals of Family Medicine. 2004; 2: 595-608.

[43] Douglasetal. The other half of the whole: teaching patients to communicate with physicians. Family Medicine, Ohio State University. 2002; 34 (5): 344-52.

[44] Hanna. Ontario Medical Association. OMA Policy on Patient centered Care. Ont Med Rev. June, 2010: 34-49.

[45] AHRQ. Agency for Healthcare Research and Quality. U.S. Department of Health and Human Services. National healthcare disparities report, 2008. March 2009.

[46] Luxfordetal. Promoting patient-centered care: a qualitative study of facilitators and barriers in healthcare organizations with a reputation for improving the patient experience. International Journal for Quality in Health Care. 2011; 23 (5): 510-5.

[47] EMPATHIE HP. Empowering patients in the management of chronic diseases. EPF, 2012.

[48] Robinson. JH. Patient-centered care and adherence: Definition and applications to improve outcomes. Journal of the American Academy of Nurse Practitioners. 2008; 20: 600-7.

[49] Tariq Saleem Alharbi ea. Implementation of person-centred care: management perspective. Journal of Hospital Administration. 2014; 3.

[50] Pelzang. R. Time to learn: understanding patient-centred care. British Journal of Nursing. 2010; 19 (14).

[51] Boyce. Conducting in-depth Interviews: A Guide for designing and conducting in-depth interviews: Pathfinder international; May 2006.

[52] Yin RK. Qualitative research from start to finish. New York, NY: Guilford Press; 2011.

[53] Alharbi TSJ. The impact of organizational culture on the outcome of hospital care: After the implementation of person-centered care. Scandinavian Journal of Public Health 2014; 42: 104-10.

[54] Mooreetal. L. Barriers and facilitators to the implementation of person-centred care in different healthcare contexts. Scand J Caring Sci. 2016. 